\title{
Rating and Ranking of Multiple-Aspect Alternatives Using Fuzzy Sets $\dagger$
}

\author{
SJOERD M. BAAS $\ddagger$ and HUIBERT KWAKERNAAK $\ddagger$
}

\author{
Fuzzy sets theory may be used to solve multiple-attribute decision problems under \\ uncertainty.
}

\begin{abstract}
Key Word Index--Decision theory; fuzzy sets; rating; multiple-attribute decision problems; multiple-objective decision problems.
\end{abstract}

\begin{abstract}
Summary-A method is proposed to deal with multiplealternative decision problems under uncertainty. It is assumed that all the alternatives in the choice set can be characterized by a number of aspects, and that information is available to assign weights to these aspects and to construct a rating scheme for the various aspects of each alternative. The method basically consists of computing weighted final ratings for each alternative and comparing the weighted final ratings. The uncertainty that is assumed to be inherent in the assessments of the ratings and weights is accounted for by considering each of these variables as fuzzy quantities, characterized by appropriate membership functions. Accordingly, the final evaluation of the alternatives consists of a degree of membership in the fuzzy set of alternatives ranking first. A practical method is given to compute membership functions of fuzzy sets induced by mappings, and applied to the problem at hand. A number of examples are worked out. The method is compared to another one proposed by Kahne who approaches the preblem probabilistically.
\end{abstract}

\section{INTRODUCTION}

IT FREQUENTLY happens that an individual, group, or community is faced with the problem of choosing among alternatives. Typically, many aspects are to be considered, to which moreover varying degrees of importance are attached. If the number of alternatives and the number of aspects are large, a very complex-looking problem may arise.

There exists a large amount of literature on this problem, dating back to the fundamental work of Fishburn [1] and earlier. Overviews of methods for multi-attribute decision making are given by MacCrimmon[2], von Winterfeld and Fischer [3], and also Roy [4].

In the present paper we restrict ourselves to a simple-minded and well-known approach, which often is resorted to. First, the aspects $a_{1}, a_{2}, \ldots, a_{n}$ that enter into the evaluation of each alternative are identified. It will be assumed that these aspects are relevant for all alternatives. The various alternatives will be

‡Department of Applied Mathematics, Twente University of Technology, Enschede, The Netherlands. denoted as $A_{1}, A_{2}, \ldots, A_{m}$. Then for a given alternative $A_{i}$, the relative merit of aspect $a_{j}$ is assessed by a rating, denoted as $r_{i j}$. Furthermore, the relative importance of each aspect is assessed by a weighting coefficient, say $w_{j}$ for aspect $a_{j}$. Then alternative $A_{i}$ receives the weighted average rating

$$
\bar{r}_{i}=\frac{\sum_{j=1}^{n} w_{j} r_{i j}}{\sum_{i=1}^{n} w_{j}} .
$$

Finally, the relevant merits of the various alternatives are judged by comparing and ranking the final ratings $\bar{r}_{1}, \bar{r}_{2}, \ldots, \bar{r}_{m}$. In this approach, it is assumed that the utilities experienced on the set of alternatives allow for numerical representations on the ordinal scale of real numbers in the sense that larger values uniquely correspond to higher utilities. Furthermore, the additive representation implies independence of the utilities involved. A theoretical approach, giving fundamental conditions for the construction of order preserving additive representation of utilities on a set of multiple-aspect alternatives is described by Fishburn[1].

Very often, this rating and ranking method is used in a situation which does not allow a more structured decision approach. Such problems are usually characterized by a lack of objective and reliable information. If in such a situation the described rating and ranking method is used, quite frequently there will be doubt and uncertainty about the exact values that are to be assigned to the various ratings and weights.

In two recent papers, Kahne [5], [6] proposes a method to account for these uncertainties. Kahne's approach is to represent the uncertainties by allowing each variable (rating or weight) to be a random variable, whose distribution is 
determined by the information that is available. Thus, for example, if the ratings assume values in the interval $[0,1]$, an assessment of 'good' for a certain aspect could be represented by assuming that the corresponding rating is a random variable, uniformly distributed on the interval $[0.7,0.9]$.

In Kahne's procedure, all weights and ratings are taken to be independent random variables, usually but not necessarily uniformly distributed. In this framework, the final ratings $\bar{r}_{1}, \bar{r}_{2}, \ldots, \bar{r}_{m}$ also become random variables. $\dagger$ In the last stage of the evaluation, the various alternatives are compared by selecting the alternative that has the highest probability of being first if the final ratings are ranked in order of descending magnitude. Kahne uses a Monte Carlo method to determine this alternative.

It is the contention of this paper that the sort of uncertainty that comes into play here is better represented by the notion of fuzziness than that of chance. The idea of fuzziness is explained in a series of papers by Zadeh, e.g. [7-9]; recently a book has appeared on the subject [10].

It is a simple exercise in the theory of fuzzy sets to reformulate Kahne's basic procedure. Section 2 of the paper is devoted to this. Kahne's ideas are somewhat elaborated by introducing measures that make it explicit how much better the preferred alternative is than the other alternatives, and how the initial uncertainties manifest themselves in the final evaluation.

In Section 3 some mathematical results are stated concerning the membership functions of fuzzy sets induced by a mapping. The proof is given in the Appendix. It is shown how this result greatly facilitates the computation of the membership functions that arise in Section 2 .

Section 4 is devoted to the presentation of a number of examples, and includes a comparison with the results of Kahne's method. Section 5 contains the conclusions of the paper.

\section{EVALUATION OF ALTERNATIVES USING FUZZY SETS}

For the basic notions of the theory of fuzzy sets we refer to Zadeh's papers. Either of the articles [7], [8] or [9] contains all the material needed in this paper. A fuzzy set will be

†Kahne uses the non-normalized weighted final rating $\bar{r}_{i}=\Sigma_{j} w_{j} r_{i j}$, where the weights do not necessarily add up to unity, contrary to the usual practice, e.g. Roy[6]. For this reason we prefer the average weighted rating (1). Using normalized weights has the desirable property that if the ratings $r_{11}, r_{12}, \ldots, r_{i n}$ all are equal, the final weighted rating $\bar{r}$ is independent of the weights and equals the common value of the rating. indicated as $\mathbf{S}=\left(X, \mu_{S}\right)$, where $X$ is the space on which the fuzzy set is defined, and $\mu_{s}(x), x \in X$, the membership function of the set. The only concepts that will be used are product fuzzy sets, fuzzy sets induced by mappings, and conditional fuzzy sets.

First, a comment is in order. The rules that will be applied in this paper for defining product fuzzy sets and composing conditional fuzzy sets are based on the minimum rule. Although a case can be made for other rules, in particular the product rule, e.g. Gaines[11], in this paper attention will be restricted to the minimum rule, which is considered in most of the literature on fuzzy sets.

As before, let $A_{1}, A_{2}, \ldots, A_{m}$ denote the alternatives that are compared and $a_{1}, a_{2}, \ldots, a_{n}$ the different aspects that the alternatives are to be judged upon. It is assumed that the fuzzy rating of aspect $a_{j}$ of alternative $A_{i}$ is represented by a membership function $\mu_{R i j}\left(r_{i j}\right)$, where $r_{i j}$ takes its values on the real line R. Similarly, the relative importance of aspect $a_{i}$ will be a fuzzy variable as well, represented by the membership function $\mu_{w_{i}}\left(w_{j}\right)$, where also $w_{j}$ takes its values on $\mathbf{R}$. All membership functions will take values in the interval $[0,1]$. Furthermore, we shall normally assume that all membership functions have finite supports, that the membership functions $\mu_{w_{j}}, j=1,2, \ldots, n$, have their supports in the positive real line, and that all membership functions assume the value 1 for at least one value of their argument.

In order to determine the fuzzy evaluation of alternative $A_{i}$ based on the fuzzy ratings and weights, consider the function $g$, mapping $R^{2 n}$ into $R$, defined by

$$
g(z)=\frac{\sum_{i=1}^{n} w_{j} r_{j}}{\sum_{j=1}^{n} w_{i}}
$$

where $z=\left(w_{1}, w_{2}, \ldots, w_{n}, r_{1}, r_{2}, \ldots, r_{n}\right)$. On the product space $\mathbf{R}^{2 n}$ we define a membership function $\mu_{z i}$, given by

$$
\mu_{Z i}(z)=\left[\bigwedge_{j=1}^{n} \mu_{w_{j}}\left(w_{j}\right)\right] \Lambda\left[\bigwedge_{k=1}^{n} \mu_{R i k}\left(r_{k}\right)\right]
$$

The symbol $\Lambda$, which is used both in prefix and infix notation, denotes the operation of taking the minimum. Through the mapping $g: \mathbf{R}^{2 n} \rightarrow \mathbf{R}$, the fuzzy set $z=\left(R^{2 n}, \mu_{Z i}\right)$ induces a fuzzy set $\overline{\mathbf{R}}_{i}=\left(\mathbf{R}, \mu_{\mathbf{R}}\right)$, with membership function

$$
\mu_{k i}(\bar{r})=\sup _{z: g(z)=f} \mu_{z i}(z), \quad \bar{r} \in \mathbf{R} .
$$


This membership function characterizes, in a fuzzy sense, the final rating of alternative $A_{i}$. The computation of $\mu_{R i}$ is discussed in Section 3 .

It is interesting to note that if the assumptions stated in the last sentence of the second paragraph of this section are satisfied, we have the property that if all aspects $a_{i}$ receive the same fuzzy ratings, the final fuzzy rating is equal to this rating, independent of the weights; compare also the footnote in Section 1. This means that if $\mu_{\text {Ri1 }}(r)=\mu_{R i 2}(r)=\ldots=\mu_{\text {Rin }}(r)=$ $\mu_{R i}(r)$ for all $r \in \mathbf{R}$, then $\mu_{\overline{R i}}(r)=\mu_{R i}(r)$ for all $r \in \mathrm{R}$. The proof of this assertion is left to the reader.

Once the membership functions $\mu_{\bar{R} i}, i=$ $1,2, \ldots, m$, of the fuzzy final ratings have been established, the question arises how to compare these ratings, and how to select the preferred alternative. Suppose for the moment that the alternatives $A_{1}, A_{2}, \ldots, A_{m}$ have received nonfuzzy final ratings $\bar{r}_{1}, \bar{r}_{2}, \ldots, \bar{r}_{m}$. Then a very plausible method to determine the preferred alternatives is to select the alternatives that have the highest final ratings. This procedure corresponds to determining the set $\left\{i \in I: \bar{r}_{i} \geqslant \bar{r}_{i}, \forall j \in I\right\}$, where $I=\{1,2, \ldots m\}$.

If the final ratings are fuzzy, this ordinary set is replaced with a fuzzy set $\left(I, \mu_{I}\right)$, whose membership function may be determined as follows. We first define the conditional fuzzy set $\left(I, \mu_{I \mid \bar{R}}\right)$, with membership function

$$
\mu_{t \mid \bar{R}}\left(i \mid \bar{r}_{1}, \bar{r}_{2}, \ldots, \bar{r}_{m}\right)=\left\{\begin{array}{c}
1 \text { if } \bar{r}_{i} \geqslant \bar{r}_{j}, \forall j \in I, \\
0 \text { otherwise }
\end{array}\right.
$$

This conditional membership function reflects that for a given combination of final ratings $\bar{r}_{1}, \bar{r}_{2}, \ldots, \bar{r}_{n}$, the $i$-th alternative belongs to the set of alternatives ranking first if and only if $\bar{r}_{i} \geqslant \bar{r}_{i}$ for all $j \in I$. Note that the conditional fuzzy set defined by this conditional membership function is not really fuzzy. Now on $\mathbf{R}^{m}$ the fuzzy final ratings define a fuzzy set $\overline{\mathbf{R}}=\left(\mathbf{R}^{m}, \mu_{\bar{R}}\right)$ with membership function

$$
\mu_{\bar{R}}\left(\bar{r}_{i}, \bar{r}_{2}, \ldots, \bar{r}_{m}\right)=\Lambda_{i=1}^{m} \mu_{\bar{R} i}\left(\bar{r}_{i}\right)
$$

The fuzzy set $\left(\mathbf{R}^{m}, \mu_{\bar{R}}\right)$ and the conditional fuzzy set $\left(I, \mu_{I \mid \mathbb{R}}\right)$ together induce a fuzzy set $\left(I, \mu_{I}\right)$ with membership function

$$
\begin{array}{r}
\mu_{I}(i)=\sup _{\bar{r}_{1}, \bar{r}_{2} \ldots \ldots \bar{r}_{m}} \mu_{|| \bar{R}}\left(i \mid \bar{r}_{1}, \bar{r}_{2}, \ldots, \bar{r}_{m}\right) \\
\Lambda \mu_{\bar{R}}\left(\bar{r}_{1}, \bar{r}_{2}, \ldots, \bar{r}_{m}\right) .
\end{array}
$$

This expression may be rewritten in the form

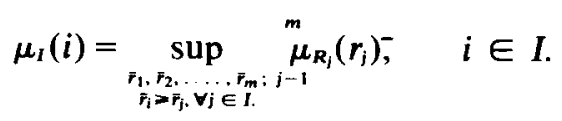

The computation of this membership function is also discussed in Section 3.

The membership function $\mu_{1}$ may be interpreted as follows. If for a given $i$ the function $\mu_{I}(i)$ assumes a certain value, say 0.8 , then this number characterizes the extent to which alternative $A_{i}$ is the best alternative.

It is noted that under the assumptions listed at the end of the second paragraph of this section, there always exists at least one alternative $A_{i}$ such that $i$ has membership 1 in the set $\left(I, \mu_{I}\right)$. This alternative corresponds to the value of $i$ which maximizes

$$
\bar{r}_{i}^{*}=\frac{\sum_{i=1}^{n} w_{i}^{*} r_{i j}^{*}}{\sum_{j=1}^{n} w_{i}^{*}}
$$

where $w_{j}^{*}$ and $r_{i j}^{*}$ are the arguments for which $\mu_{\mathrm{WJ}}$ and $\mu_{R i j}$, respectively, assume the value 1 . For simplicity we assume here that for each $i$ and $j$ there is exactly one value of the argument for which $\mu_{W_{j}}$ and $\mu_{R i j}$, respectively, assume the value 1 .

The preceding result makes it of course very simple to establish the order of preference of the alternatives, that is to say, the preference order having degree of membership one. This order of preference, together with the final ratings $\bar{r}_{i}^{*}$, $i=1,2, \ldots, n$, generating it, does not make it clear, however, whether there exist perhaps other preference orders that are almost as acceptable, in view of the uncertainty present. Such information may be obtained from the membership function $\mu_{\mathrm{l}}$.

A drawback of using the membership function $\mu_{I}$ to decide which is the best alternative is that it gives only partial information about how much better the best alternative is than the other alternatives. Also, it may happen, as indicated in the examples in Section 4, that there is more than one alternative with membership 1 in the fuzzy set $\left(I, \mu_{I}\right)$. In the case of non-fuzzy final ratings $\bar{r}_{1}, \bar{r}_{2}, \ldots, \bar{r}_{m}$, the number

$$
p_{i}=\bar{r}_{i}-\frac{1}{m-1} \sum_{\substack{i=1 \\ j \neq i}}^{m} \bar{r}_{i}
$$

where $i$ is fixed, is a measure of the preferability of alternative $A_{i}$ over the other alternatives. The expression (10) defines a mapping $h_{i}: \mathbf{R}^{m} \rightarrow \mathbf{R}$. In case the final ratings are fuzzy, the mapping $h_{i}$ 
induces a fuzzy set $P_{i}=\left(\mathbb{R}, \mu_{P_{i}}\right)$ with membership function

$$
\mu_{P i}(p)=\sup _{\substack{\bar{F}_{1}, \bar{F}_{2} \ldots \ldots, \bar{F}_{m}: \\ h_{i}\left(\bar{F}_{1}, \bar{r}_{2} \ldots, \bar{r}_{m}\right)=p}} \mu_{\bar{R}}\left(\bar{r}_{1}, \bar{r}_{2}, \ldots, \bar{r}_{m}\right), \quad p \in \mathbb{R},
$$

with $\mu_{\bar{R}}$ as defined in (6). The membership function $\mu_{P_{i}}$ may be used to judge the preferability of alternative $A_{i}$ over the other alternatives.

This section may be summarized as follows. The membership function $\mu_{\bar{R} i}$ characterizes the fuzzy final rating of alternative $A_{i}$; it follows from (4), (2), and (3). The membership function $\mu_{I}$ defines the extent to which each alternative belongs to the set of alternatives ranking first; it may be determined from (8). Finally, the membership function $\mu_{P i}$, specified by (11), (6), and (10), may be used to ascertain to what extent alternative $i$ is preferred over the other alternatives.

In Section 3, we describe how these membership functions may be evaluated. In Section 4, some examples are given illustrating the proposed method of evaluating fuzzy alternatives.

\section{EVALUATION OF MEMBERSHIP FUNCTIONS}

In the preceding section, several of the membership functions that occur are obtained according to a formula of the form

$$
\mu_{Y}(y)=\sup _{x \in \mathbb{R}^{n}: f(x)=y=y} \bigwedge_{i=1}^{n} \mu_{i}\left(x_{i}\right), \quad y \in \mathbb{R}
$$

where $\mu_{i}\left(x_{i}\right), i=1,2, \ldots, n$, are given membership functions, $f$ is a function mapping $\mathbb{R}^{n}$ into $\mathbb{R}$, and $x=\left(x_{1}, x_{2}, \ldots, x_{n}\right)$. In this section we shall state some results that will greatly facilitate the computation of the membership function $\mu_{Y}$. The first result will be given in a restricted form, which could be relaxed, at the expense, however, of a considerable loss of simplicity in the presentation. In the following it will be assumed that the membership functions $\mu_{i}, i=1,2, \ldots, n$, are piecewise continuously differentiable functions mapping $\mathbb{R}$ into $\mathbb{R}$, each bounded, nonnegative and with finite support. The function $f$ will be assumed to be a continuously differentiable mapping of $\mathbb{R}^{n}$ into $\mathbb{R}$. At the points where the respective derivatives exist, we shall denote

$$
\mu_{i}^{\prime}\left(x_{i}\right)=\frac{\mathrm{d} \mu_{i}\left(x_{i}\right)}{\mathrm{d} x_{i}}, \quad f_{i}(x)=\frac{\partial f\left(x_{1}, x_{2}, \ldots, x_{n}\right)}{\partial x_{i}}
$$

Theorem 1. Suppose that the point $\hat{x}=$ $\left(\hat{x}_{1}, \hat{x}_{2}, \ldots, \hat{x}_{n}\right) \in \mathbb{R}^{n}$ satisfies the following conditions: (i) The derivatives $\mu_{i}^{\prime}\left(\hat{x}_{i}\right)$ and $f_{i}(\hat{x}), \quad i=$ $1,2, \ldots, n$, all exist, and are all nonzero.

(ii) $\mu_{1}\left(\hat{x}_{1}\right)=\mu_{2}\left(\hat{x}_{2}\right)=\ldots=\mu_{n}\left(\hat{x}_{n}\right)$.

(iii) $\mu_{i}^{\prime}\left(\hat{x}_{i}\right) / f_{i}(\hat{x})$ has the same sign for each $i \in\{1,2, \ldots, n\}$.

Then $\hat{x}$ is a strict relative maximum point of the mathematical programming problem

$$
\operatorname{maximize} \Lambda_{i=1}^{n} \mu_{i}\left(x_{i}\right) \text {, subject to } f(x)=\hat{y}
$$

where

$$
\hat{y}=f(\hat{x})
$$

The proof of this theorem is given in the Appendix. Before stating the second theorem, we define the number $M$ and the integer set $L$ as follows

$$
M=\sup _{x \in \mathbf{R}^{n}} \bigwedge_{i=1}^{n} \mu_{i}\left(x_{i}\right), \quad L=\left\{i: \sup _{x_{i} \in \mathbb{R}} \mu_{i}\left(x_{i}\right)=M\right\} .
$$

We then have the following result, which may be used in determining the values of $y$ where the membership function $\mu_{Y}(y)$ assumes its maximum.

Theorem 2. Suppose that the point $\hat{x}=$ $\left(\hat{x}_{1}, \hat{x}_{2}, \ldots, \hat{x}_{n}\right) \in \mathbb{R}^{n}$ satisfies the following conditions:

$$
\begin{aligned}
& \mu_{i}\left(\hat{x}_{i}\right)=M \text { for each } i \in L . \\
& \mu_{i}\left(\hat{x}_{i}\right) \geqslant M \text { for each } i \notin L .
\end{aligned}
$$

Then $\hat{x}$ is a global maximum point of the mathematical programming problem

$$
\operatorname{maximize} \bigwedge_{i=1}^{n} \mu_{i}\left(x_{i}\right) \text {, subject to } f(x)=\hat{y},
$$

where

$$
\hat{y}=f(\hat{x})
$$

Also this theorem is proved in the Appendix. We finally state the following result, which may be used to establish the regions where the membership function $\mu_{Y}(y)$ assumes the value zero.

Theorem 3. Let $S \subset \mathbb{R}^{n}$ denote the support of the joint membership function $\Lambda^{n} \mu_{i}\left(x_{i}\right)$, i.e., $S=\left\{x \in \mathbf{R}^{n}: \mu_{i}\left(x_{i}\right)>0, i=1,2, \ldots, n\right\}$. Define

$$
y_{\min }=\inf _{x \in S} f(x), \quad y_{\max }=\sup _{x \in S} f(x) .
$$

Then if $\mu_{Y}$ is defined as in (12), $\mu_{Y}(y)=0$ if $y<y_{\min }$ or $y>y_{\max }$. If each of the supports of the membership functions $\mu_{i}, i=1,2, \ldots, n$, is an interval, $\mu_{Y}(y)>0$ for $y_{\min }<y<y_{\max }$. Again we refer to the Appendix for the proof. 
We shall now demonstrate how to use Theorem 1 in computing membership functions of the form (12). Suppose that we wish to determine the value or values of $y$ for which $\mu_{Y}(y)$ assumes a given value, say $\mu_{0}$. Let $\left(\hat{x}_{1}, \hat{x}_{2}, \ldots, \hat{x}_{n}\right)$ be the point for which the supremum in (12) is assumed. We shall look for such points satisfying condition (ii) of Theorem 1. Let us therefore determine for each $i$ the values of $x_{i}$ for which $\mu_{i}\left(x_{i}\right)=\mu_{0}$ as illustrated in Fig. 1. Suppose that $\mu_{i}^{\prime}\left(x_{i}\right) \neq 0, i=1,2, \ldots, n$, in all the points thus obtained, situation (a) of Fig. 1. Let $L_{i}=\left\{x_{i}: \mu_{i}\left(x_{i}\right)=\mu_{0}\right\}, i=1,2, \ldots, n$.

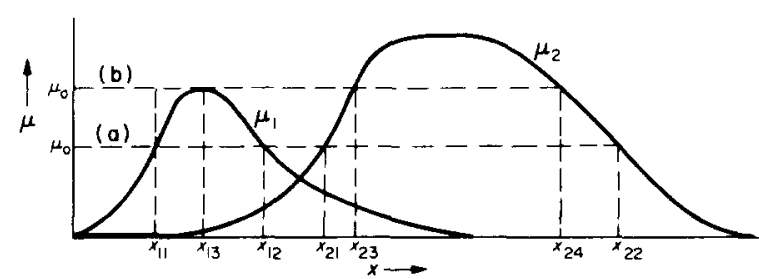

FIG. 1. Determination of membership functions. (a) Situation corresponding to Theorem $1 . L_{1}=\left\{x_{11}, x_{12}\right\}, L_{2}=\left\{x_{21}, x_{22}\right\}$. (b) Situation corresponding to Theorem 2. $L=\{1\}, L_{1}=\left\{x_{13}\right\}$, and $L_{2}=\left\{x: x \in\left[x_{23}, x_{24}\right]\right\}$.

Now determine all combinations of points $\hat{x}_{1}, \hat{x}_{2}, \ldots, \hat{x}_{n}$, with $\hat{x}_{i} \in L_{i}, i=1,2, \ldots, n$, such that the first and third requirements of Theorem 1 are satisfied. Substitution of each of these combinations of points into $f$ gives us the values of $\bar{y}=f\left(\hat{x}_{1}, \hat{x}_{2}, \ldots, \hat{x}_{n}\right)$ for which the mathematical programming problem considered has $\left(\hat{x}_{1}, \hat{x}_{2}, \ldots, \hat{x}_{n}\right)$ as a strict relative maximum point.

Theorem 2 applies in case $\mu_{0}=M$, situation (b) of Fig. 1. Then for each $i$ we determine the set $L_{i}$ defined by

$$
L_{i}= \begin{cases}\left\{x_{i}: \mu_{i}\left(x_{i}\right)=M\right\} & \text { if } i \in L, \\ \left\{x_{i}: \mu_{i}\left(x_{i}\right) \geqslant M\right\} & \text { if } i \notin L .\end{cases}
$$

Then we can determine the values of $\hat{y}$ for which $\mu_{Y}(\hat{y})=\mu_{0} \quad$ by the substitution $y=$ $f\left(\hat{x}_{1}, \hat{x}_{2}, \ldots, \hat{x}_{n}\right)$, where $\hat{x}_{i} \in L_{i}, i \in\{1,2, \ldots, n\}$. The application of Theorem 3 to determine the regions where $\mu_{Y}(y)=0$ is straightforward.

Since Theorem 1 only gives sufficient conditions for relative maximum points, the danger exists that the values of $\hat{y}$ obtained by the method described above do not satisfy $\mu_{Y}(\hat{y})=$ $\mu_{0}$. It seems difficult to establish sufficiently general conditions that exclude this possibility, however.

The method as described finds a very simple application in the calculation of the membership function of the variable

$$
p_{i}=x_{i}-\frac{1}{m-1} \sum_{\substack{j=1 \\ j \neq i}}^{m} x_{i}
$$

where the membership functions $\mu_{i}$ of the variables $x_{i}, i=1,2, \ldots, m$, are given. This is a problem we met in the preceding section. In this case we have for the partial derivatives $f_{j}$, $\mathrm{j}=1,2, \ldots, m$,

$$
f_{j}=\left\{\begin{array}{cc}
-[1 /(m-1)] & \text { for } j \neq i \\
1 & \text { for } j=i
\end{array}\right.
$$

This means that in order to compute the values of $p_{i}$ for which $\mu_{P i}\left(p_{i}\right)=\mu_{0}$, with $\mu_{0}$ a given number, we have to find numbers $\hat{x}_{1}, \hat{x}_{2}, \ldots, \hat{x}_{m}$ such that $\mu_{1}\left(\hat{x}_{1}\right)=\mu_{2}\left(\hat{x}_{2}\right)=\ldots=\mu_{m}\left(\hat{x}_{m}\right)=\mu_{0}$, and such that $\mu_{j}^{\prime}\left(\hat{x}_{i}\right), j=1,2, \ldots, m$, with $j \neq i$, all have the same signs, while $\mu_{i}^{\prime}\left(\hat{x}_{i}\right)$ has the opposite sign. This is a very simple task. Once such a combination $\hat{x}_{1}, \hat{x}_{2}, \ldots, \hat{x}_{m}$ has been found, the number $\hat{p}_{i}$ such that $\mu_{P i}\left(\hat{p}_{i}\right)=\mu_{0}$ follows by substituting the numbers $\hat{x}_{1}, \hat{x}_{2}, \ldots, \hat{x}_{m}$ into (14). Points satisfying Theorem 2 are even easier to find.

It is slightly more complicated to determine the membership function of the variable

$$
\bar{r}=\frac{\sum_{i=1}^{n} w_{i} r_{i}}{\sum_{j=1}^{n} w_{j}}
$$

where the membership functions $\mu_{W i}$ and $\mu_{R i}$ of the variables $w_{i}$ and $r_{i}, i=1,2, \ldots, n$, respectively, are given. To apply the search procedure corresponding to Theorem 1 we need the partial derivatives $f_{w j}$ of the function defined by (16) with respect to $w_{j}$ and the partial derivatives $f_{r}$ with respect to $r_{j}$. We have

$$
f_{r j}(r, w)=\frac{w_{j}}{\sum_{i=1}^{n} w_{i}}, \quad f_{w j}(r, w)=\frac{r_{j}-\bar{r}}{\sum_{i=1}^{n} w_{i}}
$$

both for $\mathrm{j}=1,2, \ldots, n$, where $r$ and $w$ denote the vectors $\left(r_{1}, r_{2}, \ldots, r_{n}\right)$ and $\left(w_{1}, w_{2}, \ldots, w_{n}\right)$, respectively, and $\bar{r}$ is given by (16). In the cases we consider, the weights $w_{j}$ are always positive, so that also $f_{r}$ is always positive. Thus, in order to compute the values of $\bar{r}$ for which $\mu_{k}(\bar{r})=\mu_{0}$, with $\mu_{0}$ a given number, we have to find numbers $\hat{r}_{1}, \hat{r}_{2}, \ldots, \hat{r}_{n}$ and $\hat{w}_{1}, \hat{w}_{2}, \ldots, \hat{w}_{n}$, such that $\mu_{R i}\left(\hat{r}_{i}\right)=$ $\mu_{w_{i}}\left(\hat{w}_{i}\right)=\mu_{0}$ for $i=1,2, \ldots, n$, and such that $\mu_{k i}^{\prime}\left(\hat{r}_{i}\right)$ and $\mu_{w_{i}}^{\prime}(\hat{w}) /\left(\hat{r}_{i}-\bar{r}\right)$ all have the same signs. The problem here is that the signs of latter quantities can only be established after all numbers $\hat{r}_{1}, \hat{r}_{2}, \ldots, \hat{r}_{n} \hat{w}_{1}, \hat{w}_{2}, \ldots, \hat{w}_{n}$ have been determined. It is not difficult, however, to envisage trial and error methods where the critical numbers $\hat{r}_{i}$, i.e., those close to $\bar{r}$, are first chosen tentatively and later adjusted. The case 
$n=2$ is particularly easy, because no matter what $\hat{w}_{1}$ and $\hat{w}_{2}$ are, one has $\hat{r}_{1} \geqslant \bar{r}$ and $\hat{r}_{2} \leqslant \bar{r}$ if $\hat{r}_{1}>\hat{r}_{2}$, and vice-versa if $\hat{r}_{1}<\hat{r}_{2}$.

We conclude this section by considering the problem of finding the membership function $\mu_{t}$ introduced in the preceding section, given by ( 8 ). The theorems are of no avail here. The membership function may systematically be determined as follows. For the given value of $i$, determine the functions

$$
\hat{u}_{i k}(r)=\left\{\begin{array}{cc}
\sup _{r_{k} \leqslant r} \mu_{\bar{R} k}\left(r_{k}\right) & \text { for } k=1,2, \ldots, m, k \neq i \\
\mu_{i}(r) & \text { for } k=i,
\end{array}\right.
$$

and

$$
\hat{\mu}_{i}(r)=\min _{k} \hat{\mu}_{i k}(r)
$$

both for $r \in \mathbb{R}$. Then

$$
\mu_{i}(i)=\sup _{r} \hat{\mu}_{i}(r)
$$

\section{EXAMPLES AND COMPARISON WITH KAHNE'S METHOD}

In this section a number of examples of applications of the method will be given. One of these examples will also be solved according to Kahne's method [5], [6]. The results of the two methods will be compared.

Example 1. For the first two examples we take two simple, related problems, where it is easy to interpret the results. We first consider a twoalternative, two-criterion problem, with ratings and weights as listed in Table 1 . Inspection of the entries shows that most likely alternative 1 will be favored. Figure 2 gives the membership functions assigned to the ratings 'good' and 'fair', while Fig. 3 gives those for the weights 'very important' and 'rather unimportant'.

Using the method outlined in the preceding section, it is not difficult to compute the membership function of the final ratings for the alternatives 1 and 2 . The resulting membership functions $\mu_{R_{1}}$ and $\mu_{R_{2}}$ are sketched in Fig. 4. It is

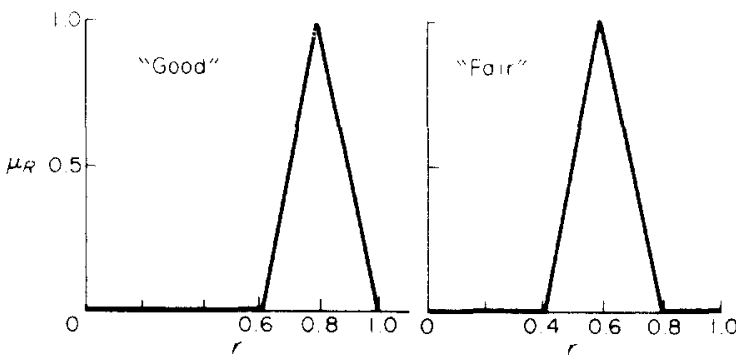

FIG. 2. Membership functions for the ratings 'good' and 'fair'

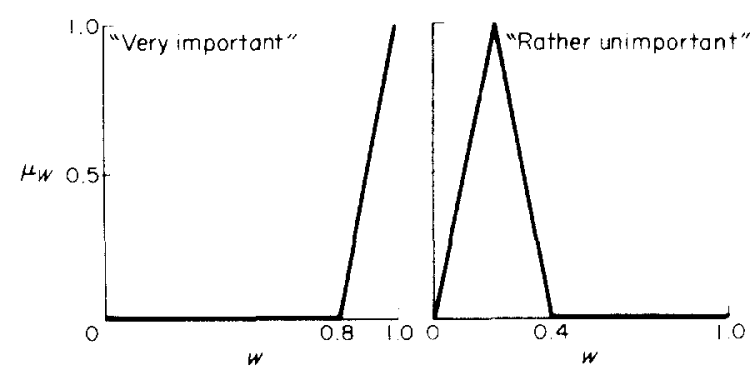

FIG. 3. Membership functions for the weights 'very important' and 'rather unimportant'.

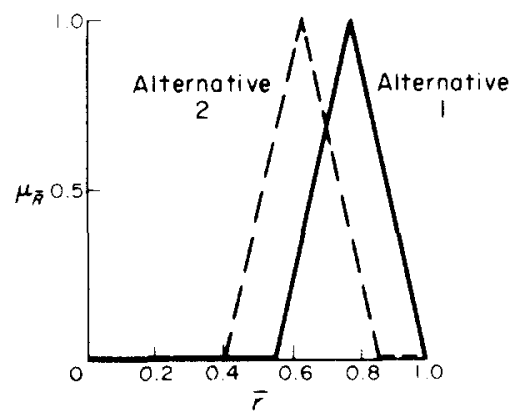

FIG. 4. Example 1: Membership functions of final ratings.

noted that the triangular shapes of the membership functions are more or less preserved.

A comparison of the membership functions of the final ratings shows that on the whole alternative 1 rates higher than alternative 2 . This is confirmed by calculating the membership function $\mu_{I}$, which is given in Table 2 . It is seen that the degree of membership of alternative 1 in the set of alternatives ranking first is 1 , but that alternative 2 still has a degree of membership 0.7 in the same set. Fig. 5 shows the membership function of the preferability $p_{1}=\bar{r}_{1}-\bar{r}_{2}$ of alternative 1 over alternative 2 . This figure confirms that alternative 1 is generally but not universally preferred over alternative 2 .

TABle 1. RATINGS AND WEIGHTS FOR EXAMPLE $\mathbf{I}$

\begin{tabular}{lccc} 
& weight & $\begin{array}{c}\text { ratings for } \\
\text { alternative } ~\end{array}$ & $\begin{array}{c}\text { ratings for } \\
\text { alternative 2 }\end{array}$ \\
\hline criterion 1 & $\begin{array}{c}\text { very } \\
\text { inportant }\end{array}$ & good & fair \\
criterion 2 & $\begin{array}{c}\text { rather } \\
\text { unimportant }\end{array}$ & fair & good \\
\hline
\end{tabular}


TABLE 2. EXAMPLE 1: THE MEMBERSHIP FUNCTION $\mu_{t}$

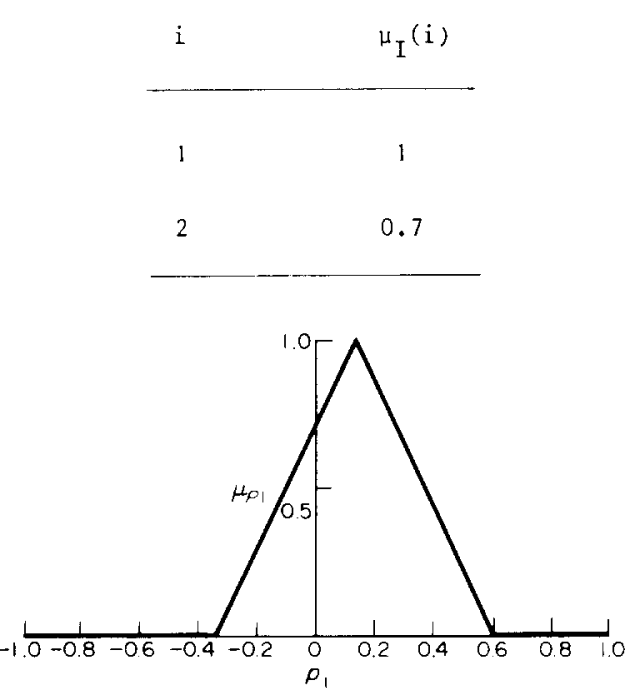

FIG. 5. Example 1: Membership function of preferability of alternative 1 over alternative 2 .

Example 2. The second example is a perturbation of Example 1, as indicated in Table 3. The only difference with Example 1 is that the rating of criterion 1 for alternative 1 has been changed from 'fair' to 'not clear'. It is obvious that this rating will make it difficult to obtain a clear-cut comparison of the alternatives. The membership function of the rating 'not clear' is indicated in Fig. 6. It is seen that the interpretation of this rating is that every rating from 0 to 1 is equally acceptable. Figure 7 gives a comparison of the membership functions of the final ratings of the two alternatives. Table 4 shows that in this case both alternatives are full members of the set of alternatives ranking first, which means that either alternative is equally

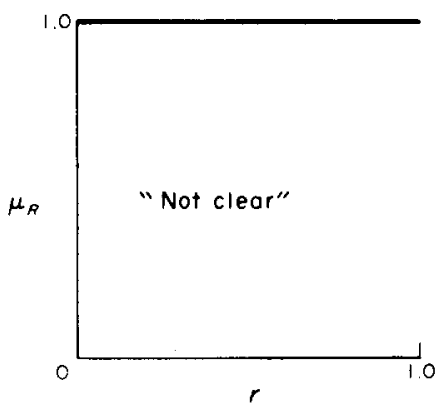

FIG. 6. Membership function for the rating 'not clear'.

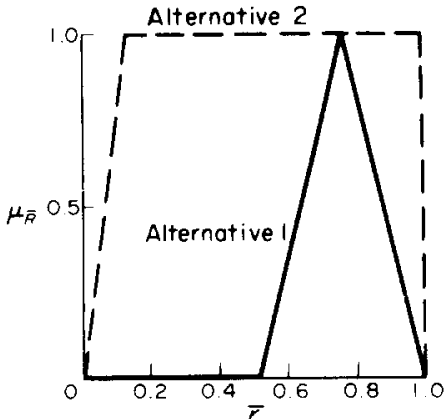

FIG. 7. Example 2: Membership functions of the final ratings of alternative 1 and 2 .

TABle 4. EXample 2: The MEMBERShip FunCtion $\mu_{4}$

$\begin{array}{cc}i & \mu_{I}(i) \\ 1 & 1 \\ 2 & 1\end{array}$

acceptable as the preferred alternative. Figure 8 shows the membership function of the preferability $p_{1}=\bar{r}_{1}-\bar{r}_{2}$ of alternative 1 over alternative 2 , which confirms the prevailing indifference.

Example 3. This example is included to illustrate the effect of choosing different shapes of the membership functions assigned to the fuzzy ratings and weights. We consider the twoalternative, two-criterion problem of Example 1, with the ratings and weights as indicated in Table 1. The uncertainties now are represented by rectangular membership functions, however, with the same supports as the triangular membership functions of Figs. 2 and 3 .

Figure 9 displays the resulting membership

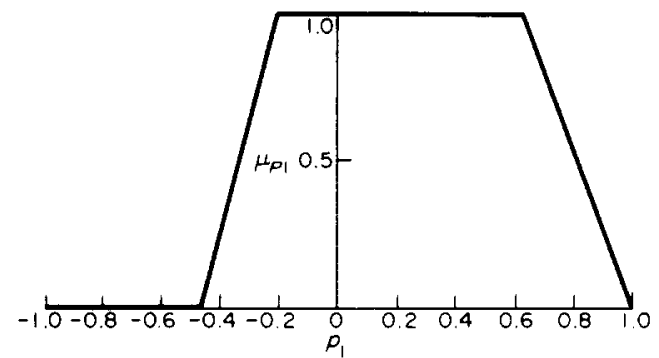

FIG. 8. Example 2: Membership function of the preferability of alternative 1 over alternative 2 .

TABle 3. RATINGS AND Weights FOR EXAMPLE 2

\begin{tabular}{lccc} 
& weight & $\begin{array}{c}\text { ratings for } \\
\text { alternative } 1\end{array}$ & $\begin{array}{c}\text { ratings for } \\
\text { alternative } 2\end{array}$ \\
\hline criterion 1 & $\begin{array}{c}\text { very } \\
\text { important }\end{array}$ & good & not clear \\
\hline criterion 2 & $\begin{array}{c}\text { rather } \\
\text { unimportant }\end{array}$ & fair & good
\end{tabular}




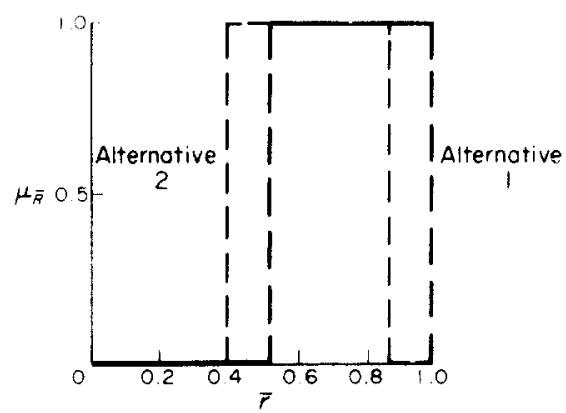

Fig. 9. Example 3: Membership functions of the final ratings of alternatives 1 and 2 .

functions of the final ratings of the two alternatives. These membership functions are again rectangular, with the same supports as the, approximately triangular, membership functions of Fig. 4. The membership of both alternatives in the set of alternatives ranking first is 1 , which does not help much in deciding which alternative is better.

Figure 10 gives the membership function of the preferability $p_{1}=\bar{r}_{1}-\bar{r}_{2}$ of alternative 1 over alternative 2 . This graph indicates that alternative 1 has advantages over alternative 2 , but this indication is far less strong than in the case of the membership function sketched in Fig. 4. It appears that the consistent use of rectangular membership functions gives rise to considerable indecisiveness at the final evaluation stage. This example also shows that the results of the analysis are sensitive to the choice of the $a$ priori membership function.

Example 4. This example is a problem with more alternatives and more criteria. Table 5 gives the various weights and ratings. A cursory inspection does not make it immediately clear which is the preferred alternative. Figs. 11 and 12 depict the membership functions of the weight 'moderately important' and the ratings 'very good', 'fair to good', and 'poor'. Figure 13 gives the resulting membership functions of the

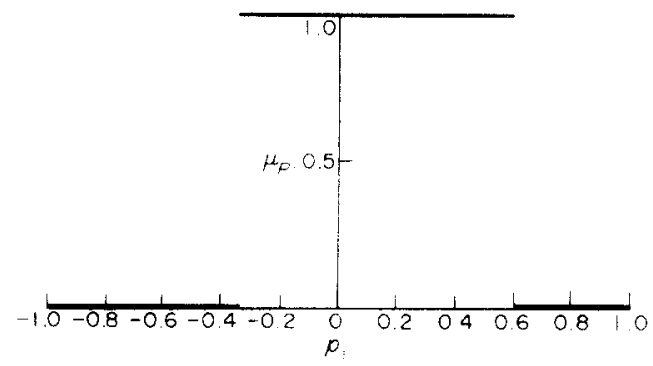

FIG. 10. Example 3: Membership function of the preferability of alternative 1 over alternative 2 .
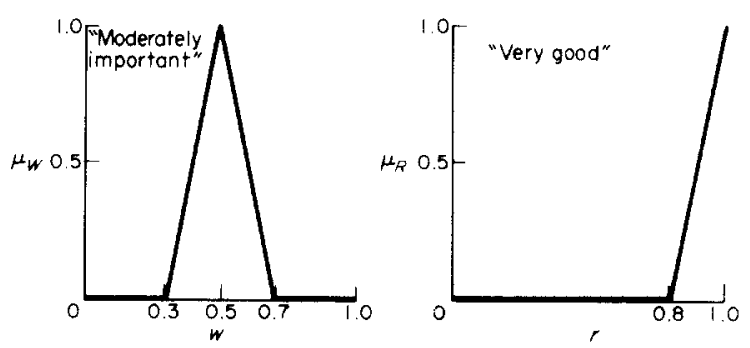

FIG. 11. Membership functions of the weight 'moderately important' and the rating 'very good'.

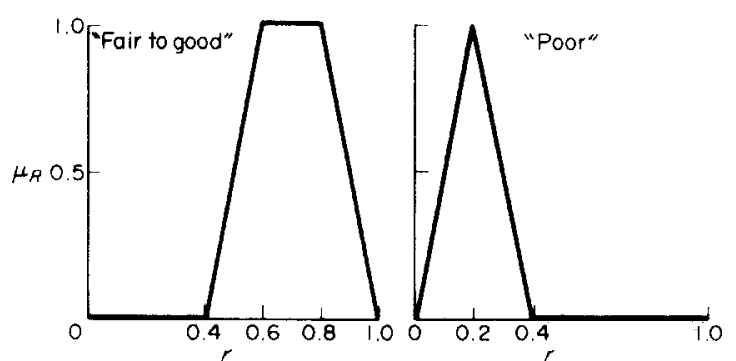

FiG. 12. Membership functions of the ratings 'fair to good' and 'poor'.

final ratings. The memberships of the alternatives in the set of alternatives ranking first appear in Table 6. We observe that alternative 2 is the preferred alternative, closely followed by alternative 1 . The membership function of the preferability $p_{2}=\bar{r}_{2}-\frac{1}{2}\left(\bar{r}_{1}+\bar{r}_{3}\right)$ of alternative 2 over the other alternatives is sketched in Fig. 14. It

TABII: 5. RATINGS AND WEIGHTS FOR EXAMPLE 4

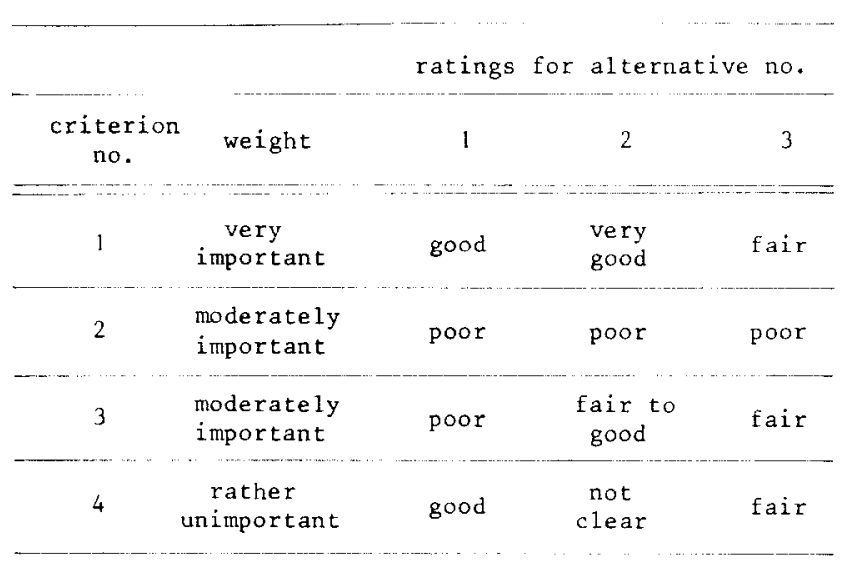




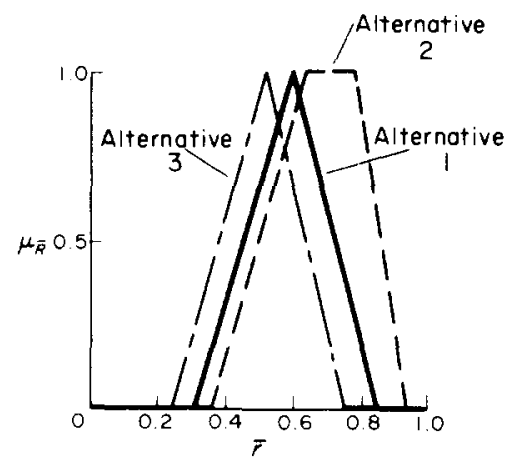

Fig. 13. Example 4: Membership functions of the final ratings.

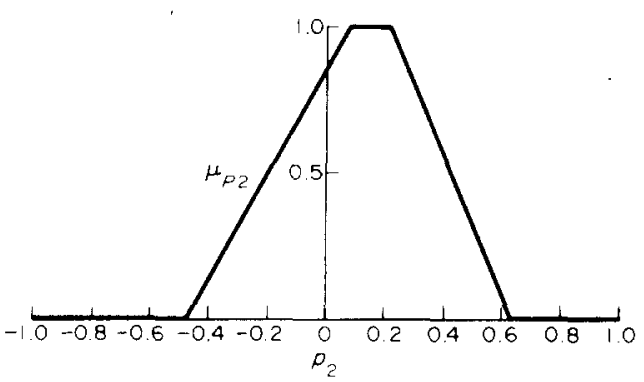

FIG. 14. Example 4: Membership function of the preferability of alternative 2 over alternatives 1 and 3 .

TABle 6. EXAmple 4: THF MEMBERSHIP FUNCTION $\mu_{1}$

\begin{tabular}{cc}
\hline$i$ & $\mu_{I}(i)$ \\
1 & 0.95 \\
2 & 1 \\
3 & 0.77 \\
\hline
\end{tabular}

is seen that alternative 2 is better, but not all that better, than the other alternatives.

Application of Kahne's method to example 4. In order to compare the present method to that of Kahne [5], [6], the problem of Example 4 has also been evaluated according to Kahne's method. The only deviation from Kahne's proposal is that the normalized final rating (1) has been employed rather than a non-normalized weighted final rating. All weights and ratings were taken to be random variables that are uniformly distributed with the same supports as the corresponding membership functions. The final ratings of the alternatives were computed according to the Monte Carlo method suggested by Kahne. This means that for each alternative, the various weights and ratings were sampled independently according to their respective probability distributions. From the sampled values, the final ratings were computed. Histograms were made of the final ratings, and a record was kept of the number of times each alternative ranked first. Each final rating was sampled 500 times. Figure 15 gives the histograms of the final ratings. It is seen that although the supports of the density functions of the final ratings are the same as those of the corresponding membership functions in Fig. 13, the histograms taper off rather quickly at the higher end, resulting in a certain bias of the final ratings towards the lower side. This phenomenon was also noticed by Kahne. It is furthermore observed that the histograms, like the membership functions, show a considerable overlap.

Table 7 displays the frequencies with which each alternative ranked first. It is seen that the probability of ranking first is much greater for alternative 2 than for the other alternatives. Accordingly, Kahne's method gives a much

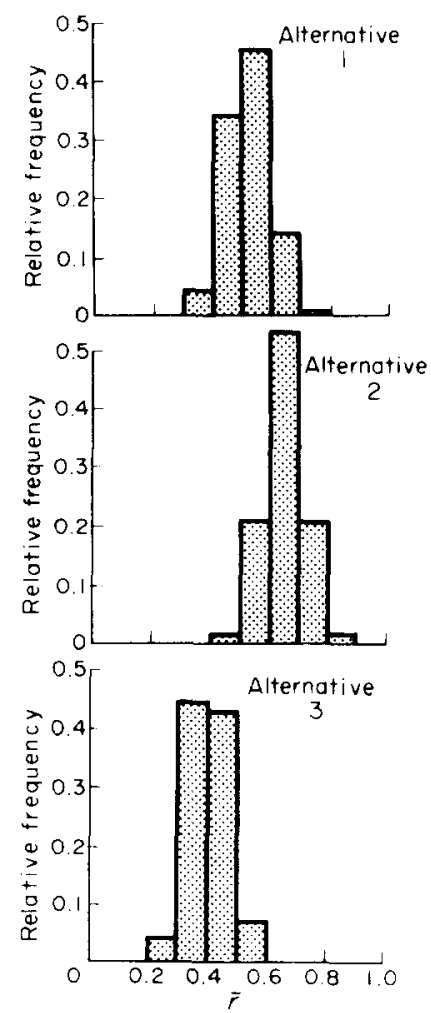

FIG. 15. Example 4: Histograms of the final ratings of alternatives 1,2, and 3 according to Kahne's method.

TABle 7. EXAMPle 4: FReQUencies WITH WHICH AlterNATIVES 1, 2, AND 3 RANKED FIRST ACCORDING TO KAHNF'S METHOD

\begin{tabular}{lc} 
& $\begin{array}{c}\text { Erequency with which } \\
\text { alternative } i \\
\text { ranked first }\end{array}$ \\
\hline 1 & 0.104 \\
2 & 0.844 \\
3 & 0.052
\end{tabular}


stronger indication of the preferability of alternative 2 over the other alternatives than the method proposed in the present paper.

\section{DISCUSSION AND CONCLUSIONS}

In this paper, a method has been proposed to deal with multiple-aspect decision making in the presence of uncertainty. The method is based on a straightforward rating and ranking method, where the weights and ratings, however, are represented as fuzzy variables. The method should be compared with a probabilistic method proposed by Kahne [5], [6]. We present a comparative discussion of the two methods.

Before doing this, it is desirable to clarify the difference between Kahne's and the present method, since it is known that fuzzy set theory may also be given a probabilistic interpretation, e.g. Gaines [11], Watanabe[12]. To make the distinction clear, we shall describe two different models, the first of which gives rise to Kahne's method, while the second results in the fuzzy sets method.

In both models we shall postulate a group of people, denoted as the committee, dealing with the decision problem at hand. In the first model, we shall assume that individual committee members are questioned about the weights $w_{i}$ and the ratings $r_{i j}$ that have to be assigned to the various attributes and alternatives. The committee members respond by stating numbers that in their opinion represent the weight or rating they are being interviewed about. These numbers are produced randomly, in agreement with the probability distributions imposed in Kahne's approach. Many committee members are interviewed, who each time respond with statistically independent numbers for the various weights and ratings. Whenever a complete set of weights and ratings for a given alternative is obtained, the corresponding final rating is calculated. Finally, statistics are compiled on the frequency of occurence of final ratings and their relative order.

This is the model for Kahne's method. Note that committee members are obliged to produce definite numbers representing the weights and ratings, although the choice of the particular number may be random.

In the second model (Gaines [13]) which will be used for the fuzzy sets method, a different procedure is envisaged. Consider for instance one of the weights, $w_{j}$. We shall postulate that the degree of membership $\mu_{w_{j}}\left(w_{j}\right)$ of a particular value $w_{j}$ for the $j$-th weight is determined as the fraction of the committee that is willing to accept this value $w_{j}$ as a possibly correct value.
Thus, $\mu_{w_{j}}\left(w_{j}\right)$ may be established by taking a poll. This procedure is repeated for all possible values of $w_{i}$, resulting in an experimentally established membership function $\mu_{w_{j}}$. All membership functions $\mu_{w_{i}}$ and $\mu_{R i j}$ occurring in the problem may be obtained in this fashion. The data thus acquired are processed according to the formalism of Section 2 of this paper, which may be explained as follows.

For a given set of weights $w_{1}, w_{2}, \ldots, w_{n}$ and ratings $r_{i 1}, r_{i 2}, \ldots, r_{i n}$ (with $i$ fixed) the degree of membership that is assigned to the joint occurrence of these numbers according to (3) is

$$
\left[\bigwedge_{j=1}^{n} \mu_{W_{j}}\left(w_{j}\right)\right] \Lambda\left[\Lambda_{j-1}^{n} \mu_{R i j}\left(r_{i j}\right)\right]
$$

This means that the joint occurrence is acceptable to the full extent to which the least acceptable individual occurrence is acceptable. Gaines terms this fuzzy logic [11]. An alternative approach would be to assume statistical independence of the individual occurrences, and assign the degree of membership

$$
\prod_{j=1}^{n} \mu_{w_{j}}\left(w_{j}\right) \prod_{j=1}^{n} \mu_{R i j}\left(r_{i j}\right)
$$

to the joint occurrence, which perhaps is not unreasonable. This approach, endowed with the name stochastic logic by Gaines[11], has not been pursued in this paper, however.

We can interpret the number (21) as the largest fraction of the committee that ever can be found to endorse the combination $w_{1}, w_{2}, \ldots, w_{n}$, $r_{i 1}, r_{i 2}, \ldots, r_{i n}$. In a similar vein,

$$
\mu_{Y i}(y)=\sup _{\substack{w_{1}, w_{2}, \ldots . w_{n} \\ r_{1}, r_{2} \ldots, r_{n}: \\ y=\frac{\sum w_{1} r_{j}}{\sum w_{j}}}}\left[\Lambda_{j=1}^{n} \mu_{W_{j}}\left(w_{j}\right)\right] \Lambda\left[\Lambda_{i=1}^{n} \mu_{R i j}\left(r_{i}\right)\right]
$$

may be interpreted as the greatest lower bound on the largest fraction of the committee that can be recruited to endorse $y$ as the final rating for alternative $A_{i}$.

It is hoped that these two models bring out the difference between the two approaches. Which of the two methods is more preferable is at this point a matter of taste, more than anything else, in the absence of a more fundamental discussion. The second model, allowing the individual committee members the opportunity to express their opinions unequivocally, is slightly more to the liking of the authors.

A disadvantage of the method using fuzzy sets is that owing to the possibilistic nature of the analysis, which has to leave room for each 
alternative, a much weaker indication is obtained of the preferability of one alternative over the others. In the case of Example 2, for instance, the present method indicates no difference in preference for the alternatives 1 and 2 , while the probabilistic method would distinctly favor alternative 1 . Also in the case of Example 4, the probabilistic method identifies alternative 2 as the preferred alternative much more positively than the fuzzy sets method. Looking over the ratings and weights as listed in Table 5, however, one wonders whether the distinction between the three alternatives is all that clear-cut.

An advantage of the method using the concept of fuzziness is that it does not suffer from the biasing effect noticed by Kahne. It appears doubtful, however, that this biasing effect seriously affects the outcome of the final conclusion.

The fuzzy set method furthermore has the property that if rectangular membership functions are assumed for the ratings and weights, the final rating also has a rectangular membership function. In the probabilistic approach, uniform distributions of the ratings and weights result in non-uniform distributions of the final ratings. Thus, in the latter case, 'uniform uncertainty' in the initial data does not result in uniform uncertainty in the final result.

Example 3 of Section 5 demonstrates that in the fuzzy sets approach, the results of the analysis are sensitive to the $a$ priori choice of the membership functions. This is a common phenomenon in applications of fuzzy sets theory, and is often used to criticize the theory. The 'committee paradigm' for fuzzy sets theory, as described earlier in this section, may help to resolve this problem.

Finally, an advantage of the method using fuzzy weightings and ratings is that it is computationally attractive as compared to Kahne's method. Problems involving moderate numbers of alternatives and aspects can easily be solved by hand, while larger problems can be dealt with through the use of a computer. The Monte Carlo method of Kahne, although in terms of programming certainly much simpler than the present approach, normally requires the use of a computer also for small problems, involving substantially larger amounts of computer time.

In conclusion, it appears that both the method presented here and that proposed by Kahne may be useful in tackling multiple objective decision problems under uncertainty. Both methods have as characteristic feature that not only uncertainty in the ratings and weights can be handled, but that also an indication is ob- tained to what extent the initial uncertainty is still present in the final evaluation.

Acknowledgements - The authors are pleased to acknowledge long discussions with J. P. M. Berntssen on the subject of this paper, as well as some very useful hints from Prof. R. A. Cuninghame-Green. Dr. B. R. Gaines of the University of Sussex, who read an earlier version of the paper, contributed a number of fundamental comments.

\section{REFERENCES}

[1] P. C. FISHBURN: Utility Theory for Decision Making. Wiley, New York (1964).

[2] K. R. MACCRIMMON: An overview of multiple objective decision making. In: Multiple Criteria Decision Making. J. L. Cochrane and M. Zeleny, eds. University of South Carolina Press, Columbia, S.C. (1973).

[3] D. von Winterfeld and G. W. Fischer: Multipleattribute utility theory: models and assessment procedures. In: utility, Probability and Human Decision Making. D. WENDT and CH. VLeK, eds. Reidel, Dordrecht, Netherlands (1975).

[4] B. Roy: Problems and methods with multiple objective functions. Math. Progr. 1, 239-266 (1971).

[5] S. KAHNE: A procedure for optimizing development decisions. Automatica 11, 261-269 (1975).

[6] S. KAHNE: A contribution to decision making in environmental design. Proc. IEEE 63, 518-528 (1975).

[7] L. A. ZaDEH: Fuzzy sets. Inf. Contr. 8, 338-353 (1965).

[8] R. E. BELLMAN and L. A. ZADEH: Decision making in a fuzzy environment. Manmt Sci. 17, Ser. B, nr. 4, 141164 (1970).

[9] L. A. ZADEH: Toward a theory of fuzzy systems. In: Aspects of Network and Systems Theory. R. E. KALMAN and N. DeClarIs, eds. Holt, Rinehart \& Winston, New York (1971).

[10] A. KaufmanN: Introduction à la Théorie des SousEnsembles Flous. 1. Eléments Théoretiques de Base. Masson \& Cie, Paris (1974).

[11] B. R. GAINES, Stochastic and fuzzy logics. Electron. Lett. 11, nr. 9 (1975).

[12] S. WATANABE, Creative learning and propensity automata. IEEE Trans. Systems, Man and Cybernetics 5 , nr. 6, 603-609 (1975).

[13] B. R. GaINES, Fuzzy reasoning and the logic of uncertainty. Report No. EES-MMS-UNC-75, Man-Machine System Laboratory, Dept. of Electrical Engineering, University of Essex, Colchester, U.K. (1975).

[14] J. DieudonNE, Foundations of Modern Analysis. Academic Press, New York (1960).

APPENDIX: PROOFS OF THEOREMS 1-3

In the following, the set $\{1,2, \ldots, n\}$ will be denoted as $N$. We first give an elementary proof of Theorem 1. A well-known device from nonlinear programming is employed. The problem

$$
\operatorname{maximize} \Lambda_{i=1}^{n} \mu_{i}\left(x_{i}\right) \text {, subject to } f(x)=y,
$$

is equivalent to the problem

maximize $z$, subject to $z \leqslant \mu_{i}\left(x_{i}\right), i \in N$, (A.2) and

$$
f(x)=y \text {. }
$$

In the latter problem $\left(z, x_{1}, x_{2}, \ldots, x_{n}\right)$ is the independent variable. If $(\hat{z}, \hat{x})$ solves the second problem, then $\hat{x}$ solves the first. We first study 
the second problem. Let the point $x \in \mathbb{R}^{n}$ satisfy the conditions (i), (ii), and (iii) of Theorem 1, and define $\hat{z}=\bigwedge_{i=1}^{n} \mu_{i}\left(\hat{x}_{i}\right)$. We consider the point $(z, x)=(\hat{z}+\delta z, \hat{x}+\delta x)$, and determine $\delta z \in \mathbb{R}$ and $\delta x \in \mathbb{R}^{n}$ such that the constraints $z \leqslant \mu_{i}\left(x_{i}\right)$, $i \in N$, and $f(x)=\hat{y}$ are satisfied to first order. Since according to (i) the functions $\mu_{i}$ and $f$ are differentiable at $\hat{x}$, while from (ii) $\hat{z}=\mu_{i}\left(\hat{x}_{i}\right)$, $i \in N$, and $f(\hat{x})=\hat{y}$, it follows that

$$
\begin{gathered}
\delta z \leqslant \mu_{i}^{\prime}\left(\hat{x}_{i}\right) \delta x_{i} \quad \text { for } i \in N, \\
\sum_{i=1}^{n} f_{i}(\hat{x}) \delta x_{i}=0 .
\end{gathered}
$$

Define $\delta \xi_{i}=f_{i}(\hat{x}) \delta x_{i}, \quad i \in N$. According to (i), $f_{i}(\hat{x}) \neq 0$ for each $i$, so that $\delta x_{i}=\delta \xi_{i} / f_{i}(\hat{x})$ and (A.3) and (A.4) may be rewritten in the form

$$
\begin{gathered}
\delta z \leqslant \frac{\mu_{i}^{\prime}\left(\hat{x}_{i}\right)}{f_{i}(\hat{x})} \delta \xi_{i} \quad \text { for } i \in N, \\
\sum_{i=1}^{n} \delta \xi_{i}=0
\end{gathered}
$$

If $\delta x \neq 0$, it follows from (A.6) that $\delta \xi_{j}>0$ for at least one value $j \in N$, and $\delta \xi_{k}<0$ for at least one other value of $k$ in the same set. Since from (iii) the quantity $\mu_{i}^{\prime}\left(\hat{x}_{i}\right) / f_{i}(\hat{x})$ is nonzero and does not change sign on $N$, we have either

$$
\frac{\mu_{j}^{\prime}\left(\hat{x}_{j}\right)}{f_{i}(\hat{x})} \delta \xi_{j}<0, \quad \text { or } \frac{\mu_{k}^{\prime}\left(\hat{x}_{k}\right)}{f_{k}(\hat{x})} \delta \xi_{k}<0 . \quad \text { (A.7) }
$$

From this fact and (A.5) it follows that $\delta z<0$. which shows that the point $(\hat{z}, \hat{x})$ is a strict relative maximum point of the problem (A.2). As a result, the point $\hat{x}$ satisfying the conditions of Theorem 1 is a strict relative maximum point of the problem (A.1), which proves Theorem 1.

The proof of Theorem 2 is trivial. Clearly the point $\hat{x}$ satisfies the constraint equation $f(\hat{x})=\hat{x}$. Also, $\bigcap_{i=1}^{n} \mu_{i}\left(\hat{x}_{i}\right)=M$. There can be no point $x^{*}$ satisfying the constraint equation such that $\stackrel{n}{\Lambda} \mu_{i}\left(x_{i}^{*}\right)>M$, since by definition $\stackrel{n}{\Lambda}_{\mu} \mu_{i}\left(x_{i}\right) \leqslant M$ for all $x \in \mathbb{R}^{n}$. Therefore, $\hat{x}$ is a global maximum point of the problem (A.1).

Theorem 3 is also easily proved. If $y<y_{\min }$ or $y>y_{\max }$ then any $x^{*}$ such that $f\left(x^{*}\right)=y$ is not in S. As a result $\bigwedge_{i=1}^{n} \mu_{i}\left(x_{i}^{*}\right)=0$ and also $\mu_{Y}(y)=0$. If the supports of the membership functions $\mu_{i}$, $i \in N$, are all intervals, $S$ is a connected set. Since by hypothesis $f$ is continuous for each $y$ with $y_{\min }<y<y_{\max }$ there exists according to Bolzano's theorem [14] an $x^{*} \in S$ such that $f\left(x^{*}\right)=y$. Since $\mu_{Y}(y) \geqslant \Lambda_{i}^{n} \mu_{i}\left(x_{i}^{*}\right)>0$ it follows that $\mu_{Y}(y)>0$. 